\title{
3D Real-Space Calculation of the Continuum Response
}

\author{
Takashi NakatsukasA and Kazuhiro Yabana*, \\ Physics Department, Tohoku University, Sendai 980-8578, Japan \\ * Institute of Physics, University of Tsukuba, Tsukuba 305-8571, Japan
}

(Received )

\begin{abstract}
We present that a linear response theory in the continuum can be easily formulated with Absorbing Boundary Condition (ABC). The theory is capable of describing continuum spectra and dynamical correlations. Application of the $\mathrm{ABC}$ does not require the spherical symmetry and the method is suitable for mesh representation in the real coordinate space. Isovector giant dipole resonances in beryllium isotopes are studied with the time-dependent Hartree-Fock with the Skyrme force in a three-dimensional mesh space with the ABC.
\end{abstract}

\section{$\S 1$. Introduction}

The drip-line nuclei are finite fermion systems whose separation energy is nearly zero. For such weakly bound systems, the continuum should be properly taken into account in description of their structures and reactions. In the linear response regime, the inclusion of the single-particle continuum for particle-hole $(\mathrm{p}-\mathrm{h})$ excitations has been achieved by using a method, so called, Continuum Random-Phase Approximation (CRPA). ${ }^{1)}$ The trick to treat the continuum was use of the single-particle Green's function with the Outgoing Boundary Condition (OBC). This relies on explicit calculations of Green's functions with the OBC in the one-dimensional (radial) coordinate. We have developed a method to calculate the continuum response in the three-dimensional (3D) real space by solving equations iteratively with the Green's functions. ${ }^{2)}$,3) The OBC is explicitly taken into account with this method. However, it is costly in computer resources. We have also been investigating a possibility to approximate the OBC by Absorbing Boundary Condition (ABC). ${ }^{2)-4)}$ An advantage of the $\mathrm{ABC}$ is its simplicity and flexibility. For instance, it is extremely difficult to treat the $\mathrm{OBC}$ in the real-time calculation of the time-dependent wave functions, while it is straightforward to implement the real-time method with the ABC. ${ }^{2}$ )

The Skyrme Hartree-Fock (HF) theory ${ }^{5)}$ has been extensively applied to study of ground-state properties of relatively heavy nuclei. To investigate their excited states, a straightforward extension is the linear response calculation based on the HF ground state. The CRPA combined with the Skyrme Hartree-Fock (HF) theory has been extensively utilized to study giant resonances in spherical nuclei. ${ }^{6)-8)}$ So far, only spherical nuclei were studied because of the limitation of the conventional CRPA. We would like to show that the linear response calculation with ABC is a feasible extension of the spherical CRPA to the deformed one. 


\section{§2. Skyrme Time-Dependent Hartree-Fock (TDHF) with ABC}

Since the Hamiltonian in the Skyrme HF theory is almost diagonal in coordinate representation, a mesh representation in the $3 \mathrm{D}$ coordinate space provides an economical description. The real-time calculation of the Skyrme TDHF were carried out for studies of heavy-ion reactions. ${ }^{9}$ ) The main issue is then how to incorporate the proper boundary condition in the 3D uniform grid representation. In this paper, we adopt a method of ABC combined with the real-time TDHF. ${ }^{2)}$

As is well known, the Green's function with $\mathrm{OBC}$ (for $E>0$ ) can be simply written as

$$
G^{(\mathrm{OBC})}\left(\mathbf{r}, \mathbf{r}^{\prime} ; E\right)=\left\langle\mathbf{r}\left|\frac{1}{E-H+i \eta}\right| \mathbf{r}^{\prime}\right\rangle
$$

where $\eta$ is a positive infinitesimal. In numerical calculations, we take a spherical box with radius $R$ as a model space. Since the infinitesimal $\eta$ cannot be treated directly in actual numerical calculation, we need to explicitly construct the outgoing solutions at the boundary $(r \geq R)$. This is a difficult task when the system does not possess a spherical symmetry. The idea of ABC is to replace the $\eta$ by a finite coordinate-dependent quantity, $\left\langle\mathbf{r}|\tilde{\eta}| \mathbf{r}^{\prime}\right\rangle=\tilde{\eta}(\mathbf{r}) \delta\left(\mathbf{r}-\mathbf{r}^{\prime}\right)$, and imposes the vanishing boundary condition:

$$
G^{(\mathrm{ABC})}\left(\mathbf{r}, \mathbf{r}^{\prime} ; E\right)=\left\langle\mathbf{r}\left|\frac{1}{E-H+i \tilde{\eta}}\right| \mathbf{r}^{\prime}\right\rangle
$$

Here, $\tilde{\eta}(\mathbf{r})$ is taken to be positive far outside the system $(R<r<R+\Delta r)$ and zero elsewhere. The Green's function has a vanishing boundary condition, $G^{(\mathrm{ABC})}\left(\mathbf{r}, \mathbf{r}^{\prime} ; E\right)=0$ for $r=R+\Delta r$ or $r^{\prime}=R+\Delta r$. This is equivalent to bound-state Green's function for the Hamiltonian with a complex absorbing potential, $H-i \tilde{\eta}(\mathbf{r})$. Now let us discuss properties of $G^{(\mathrm{ABC})}(E)$.

For $E<0$, the Green's function with $\mathrm{ABC}$ has a pole at eigenenergies $E=E_{n}$, because the bound eigenstate is localized, $\psi_{n}(\mathbf{r}) \approx 0$ at $r>R$. For $E>0$, the Green's function has an outgoing asymptotic behavior. This can be seen as follows: In the outer region of our model space $(r>R)$, the complex potential $-i \tilde{\eta}$ produces complex wave numbers, $k+i \gamma$ where $\gamma>0$. Thus, the outgoing waves exponentially damp while incoming waves diverge. The vanishing boundary condition selects only the outgoing solutions. In the inner model space $(r<R)$, as far as the reflection caused by the potential, $-i \tilde{\eta}(\mathbf{r})$, is negligible, $G^{(\mathrm{ABC})}(E)$ is identical to $G^{(\mathrm{OBC})}(E)$. Therefore, the complex boundary potential must be strong enough to absorb the whole outgoing wave and simultaneously gentle enough to minimize the reflection. We adopt an absorbing potential of linear dependence on the radial coordinate,

$$
\tilde{\eta}(r)= \begin{cases}0, & \text { for } r<R \\ \eta_{0} \frac{r-R}{\Delta r}, & \text { for } R<r<R+\Delta r\end{cases}
$$

In order to minimize the reflection, the height $\eta_{0}$ and width $\Delta r$ should satisfy a condition ${ }^{10), 2)}$

$$
20 \frac{E^{1 / 2}}{\Delta r \sqrt{8 m}}<\eta_{0}<\frac{1}{10} \Delta r \sqrt{8 m} E^{3 / 2}
$$


An advantage of the ABC is its applicability to the real-time TDHF calculations. We use the Skyrme TDHF method to investigate giant resonances in the continuum. The initial state at $t=0$ is prepared by boosting the HF ground state, $\left|\Psi_{0}\right\rangle$, with an instantaneous external field, $V_{\text {ext }}(\mathbf{r}, t)=v_{\text {ext }}(\mathbf{r}) \delta(t)$. Then, the time evolution of the TDHF sate, $|\Psi(t)\rangle$, in a $3 \mathrm{D}$ real space is calculated according to a prescription given in Ref. 11). Time step is taken as $\Delta t=0.001 \mathrm{MeV}^{-1}$ and $N$-times iteration of time evolution is done for $|\Psi(t)\rangle$ up to the total period $T=N \Delta t$. After the time evolution, we carry out the Fourier transform of expectation values of a onebody operator $\hat{D},\langle\Psi(t)|\hat{D}| \Psi(t)\rangle$. The imaginary part of this quantity gives the corresponding excitation strength, $S(\hat{D} ; E)=\sum_{n}|\langle n|\hat{D}| 0\rangle|^{2} \delta\left(E-E_{n}\right)$. See Ref. 2) for details of the numerical calculation.

\section{$\S 3 . \quad$ Numerical results and discussions}

\subsection{Continuum effects: an illustration}

In many cases, the RPA response functions have been calculated utilizing the $L^{2}$ basis set, such as harmonic oscillator basis. A similar $L^{2}$-type calculation is possible using the coordinate space of finite box with the vanishing boundary condition. We call this "Box Boundary Condition (BBC)". The lowenergy RPA response below the separation energy are well described with the $\mathrm{BBC}$. Then, in order to obtain a reasonable $\mathrm{BBC}$ description of the response in a energy region above the separation energy, what size of model space is required? In other words, how large the model space should be, to simulate the continuum wave functions?

Assuming the $\mathrm{BBC}$ of box radius $R$, an escaping particle of velocity $v$ (energy $E$ ) reflects at the boundary wall at $r=R$. This reflection gives a spurious contribution to the continuum response of the system and eventually produces a discrete spectrum. Thus, the maximum period of physical time evolution is

$$
T=R / v=R \sqrt{M / 2 E} .
$$

The uncertainty principle tells that the total period $T$ is related to an energy

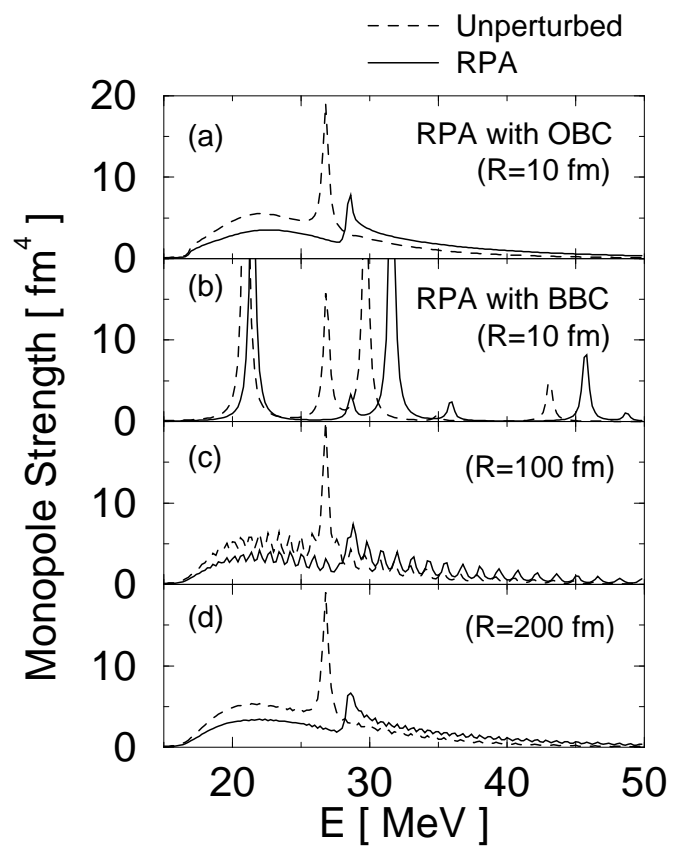

Fig. 1. Strength functions of giant monopole resonances in ${ }^{16} \mathrm{O}$ calculated with the $\mathrm{BKN}$ interaction. A smoothing parameter, $\Gamma=$ $0.5 \mathrm{MeV}$, is added to the energy as $E+$ $i \Gamma$. (a) Results of CRPA using a radial coordinate up to $R=10 \mathrm{fm}$. (b) RPA with the $\mathrm{BBC}$ of $R=10 \mathrm{fm}$. (c) The same as (b) except for $R=100 \mathrm{fm}$. (d) The same as (b) except for $R=200 \mathrm{fm}$. 
resolution $\Delta E$ after the Fourier transform:

$$
\Delta E=\frac{2 \pi}{T}=\frac{2 \pi}{R} \sqrt{\frac{2 E}{M}} .
$$

Here, $M$ and $E$ are the mass and the kinetic energy of escaping particles, respectively. Therefore, for instance, if we require $\Delta E=1 \mathrm{MeV}$ for the strength function at $E=10 \mathrm{MeV}$ above the threshold, one has to handle the box of $R \approx 200 \mathrm{fm}$. Dealing with this large space is difficult even for a spherical (1D) case and is almost impossible for a deformed (3D) case.

Using a simplified Skyrme force, the BKN interaction, ${ }^{12)}$ monopole response in ${ }^{16} \mathrm{O}$ is calculated both with and without the continuum. For this case, owing to the simplicity of the BKN interaction and the spherical symmetry of the HF state, we may carry out the RPA calculation with the $\mathrm{BBC}$ in a large coordinate space. We also perform the CRPA calculation. The OBC calculation is done for a spherical box of $R=10 \mathrm{fm}$, the result of which is compared with the BBC results with three different boxes of $R=10,100$, and $200 \mathrm{fm}$ (Fig. 1). The figure shows that we need utilize a coordinate space of $R>100 \mathrm{fm}$ to obtain a sensible result. On the other hand, if we use the complex potential of BBC, Eq. $(2 \cdot 3)$, a space of $\Delta r=10 \mathrm{fm}$ in addition to $R=10 \mathrm{fm}$ is enough to obtain an identical result to the OBC. ${ }^{4)}$

\subsection{Giant dipole resonances in beryllium isotopes}

Isovector dipole states in even beryllium (Be) isotopes $\left({ }^{8-14} \mathrm{Be}\right)$ are investigated using the real-time-ABC method in Sect.2. We adopt the SIII parameters for the Skyrme interaction including all time-even densities and most of time-odd densities.

13) Properties of the HF ground states of these isotopes were investigated in Ref. 14). The ground-state binding energies are well reproduced with the SIII and prolate shapes of the density distributions are obtained for ${ }^{8,10,14} \mathrm{Be}$. We now investigate the $E 1$ linear response on these ground states. An initial state for the real-time TDHF calculation is prepared by boosting the ground state with an $E 1$ operator with a recoil charge, $v_{\text {ext }}=(Z / A) \boldsymbol{r}_{n}-(N / A) \boldsymbol{r}_{p}$. The total period of time evolution is taken as $T=4 \mathrm{MeV}^{-1}$. This corresponds to about an energy resolution of $1.6 \mathrm{MeV}$. The model space we take is a $3 \mathrm{D}$ lattice coordinate space of $R=8 \mathrm{fm}$ with a square mesh of $\Delta x=\Delta y=\Delta z=1 \mathrm{fm}$. For the ABC potential, Eq. (2.3), we take $\eta_{0}=14 \mathrm{MeV}$ and $\Delta r=10 \mathrm{fm}$.

In Fig. 2, the obtained $E 1$ strength functions are presented. Since ${ }^{8,10,14}$ Be have prolate shapes in the ground states, the $E 1$ strengths parallel to the symmetry $(z)$ axis and perpendicular ( $x$ and $y$ ) axes are displayed separately. Large deformation splitting over $10 \mathrm{MeV}$ is obtained in these nuclei. This is because the ground states have large deformations which almost correspond to superdeformation in heavy nuclei. The ground state of ${ }^{12} \mathrm{Be}$ is nearly spherical and we show the $E 1$ response summed with respect to three directions in Fig. 2 (c).

Appearance of low-energy (pigmy) strengths $(E<10 \mathrm{MeV})$ is seen in ${ }^{12} \mathrm{Be}$ and even enhanced in ${ }^{14} \mathrm{Be}$. These strengths disappear if we get rid of the $\mathrm{ABC}$ potential, which suggests that the continuum is essential to produce the low-energy $E 1$ strengths near threshold. It is also worth noting that the real part of the response 


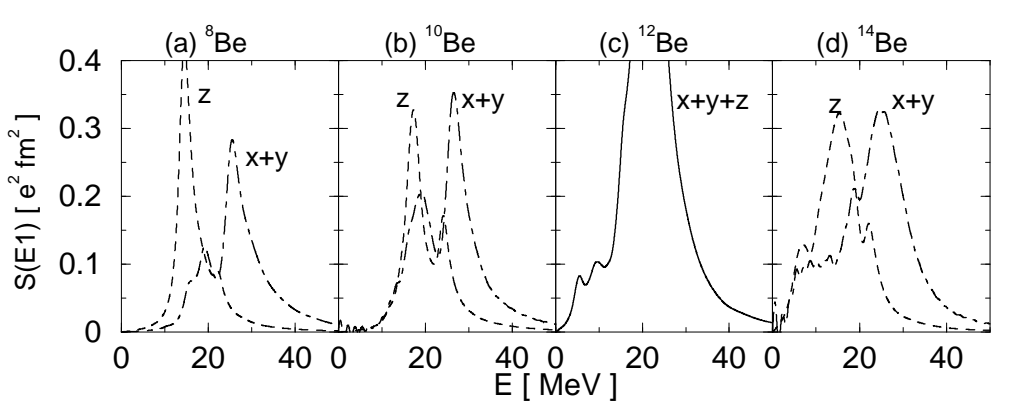

Fig. 2. $E 1$ strength functions of giant dipole resonances in even- $A$ Be isotopes, calculated by the TDHF with the SIII force using the ABC potential: $R=8 \mathrm{fm}, \Delta r=10 \mathrm{fm}, \eta_{0}=14 \mathrm{MeV}$. $E 1$ strengths parallel (perpendicular) to the symmetry axis are shown by dashed (dot-dashed) lines.

function changes its sign at the position of the low-energy peak. This may suggest a real resonance character.

\section{$\S 4$. Conclusions}

In this paper, we present the method of $\mathrm{ABC}$ which is able to describe the $3 \mathrm{D}$ continuum effects in a feasible manner and in good accuracy. We take $E 1$ giant resonances in beryllium isotopes and apply the real-time TDHF-ABC method. Large deformation splittings in ${ }^{8,10,14} \mathrm{Be}$ and low-energy resonances in ${ }^{12,14} \mathrm{Be}$ are obtained.

Although we have shown only the application to the response calculations in this paper, the $\mathrm{ABC}$ provides a general and powerful method to treat the OBC. Another application, a calculation of breakup processes of a halo nucleus, is also under progress. ${ }^{15)}$ We are encouraged by the results we have obtained and will study structures and reactions of unstable nuclei using the ABC.

\section{References}

1) S. Shlomo and G. Bertsch, Nucl. Phys. A243 (1975), 507.

2) T. Nakatsukasa and K. Yabana, J. Chem. Phys. 114 (2001), 2550.

3) T. Nakatsukasa and K. Yabana, Proceedings of the International Symposium on Physics at Drip Lines, RIKEN Review No. 39 (RIKEN, Wako, 2001), p. 96.

4) T. Nakatsukasa and K. Yabana, to be published in the proceedings of 7 th International Spring Seminar on Nuclear Physics Challenges of Nuclear Structure, Maiori, Italy, 2001, (World Scientific); Preprint nucl-th/0107023.

5) D. Vautherin and D. Brink, Phys. Rev. C5 (1972), 626.

6) N. van Giai and H. Sagawa, Nucl. Phys. A371 (1981), 1.

7) I. Hamamoto, H. Sagawa, and X. Z. Zhang, Phys. Rev. C53 (1996), 765.

8) I. Hamamoto and H. Sagawa, Phys. Rev. C53 (1996), R1492; C54 (1996), 2369.

9) J. W. Negele, Rev. Mod. Phys. 54 (1982), 913, and references therein.

10) M. S. Child, Mol. Phys. 72 (1991), 89.

11) H. Flocard, S. Koonin, and M. Weiss, Phys. Rev. C17 (1978), 1682.

12) P. Bonche, S. Koonin, and J. W. Negele, Phys. Rev. C13 (1976), 1226.

13) P. Bonche, H. Flocard, and P. H. Heenen, Nucl. Phys. A467 (1987), 115. We neglect a term of $\boldsymbol{V}_{\tau} \cdot \boldsymbol{\sigma}$ in Eq. (5) of this paper.

14) S. Takami, K. Yabana, and K. Ikeda, Prog. Theor. Phys. 94 (1995), 1011.

15) K. Yabana, contribution to this symposium. 